\title{
Summation Formulas Obtained by Means of the Generalized Chain Rule for Fractional Derivatives
}

\author{
S. Gaboury and R. Tremblay \\ Department of Mathematics and Computer Science, University of Quebec at Chicoutimi, QC, Canada G7H $2 B 1$ \\ Correspondence should be addressed to S. Gaboury; slgabour@uqac.ca
}

Received 7 May 2014; Revised 11 August 2014; Accepted 18 August 2014; Published 28 August 2014

Academic Editor: Arcadii Z. Grinshpan

Copyright (C) 2014 S. Gaboury and R. Tremblay. This is an open access article distributed under the Creative Commons Attribution License, which permits unrestricted use, distribution, and reproduction in any medium, provided the original work is properly cited.

In 1970, several interesting new summation formulas were obtained by using a generalized chain rule for fractional derivatives. The main object of this paper is to obtain a presumably new general formula. Many special cases involving special functions of mathematical physics such as the generalized hypergeometric functions, the Appell $F_{1}$ function, and the Lauricella functions of several variables $F_{D}^{(n)}$ are given.

\section{Introduction}

The fractional derivative of arbitrary order $\alpha, \alpha \in \mathbb{C}$, is an extension of the familiar $n$th derivative $D_{g(z)}^{n} F(z)=$ $d^{n} F(z) /(d g(z))^{n}$ of the function $F(z)$ with respect to $g(z)$ to nonintegral values of $n$ and is denoted by $D_{g(z)}^{\alpha} F(z)$. The aim of this concept is to generalize classical results of the $n$th order derivative to fractional order. Most of the properties of the classical calculus have been expanded to fractional calculus, for instance, the composition rule [1], the Leibniz rule [2,3], the chain rule [4], and Taylor's and Laurent's series [5-7]. Fractional calculus also provides tools that make it easier to deal with special functions of mathematical physics [8].

The most familiar representation for fractional derivative of order $\alpha$ of $f(z)$ is the Riemann-Liouville integral [9]; that is,

$$
D_{z}^{\alpha} f(z)=\frac{1}{\Gamma(-\alpha)} \int_{0}^{z} f(\xi)(\xi-z)^{-\alpha-1} d \xi,
$$

which is valid for $\Re(\alpha)<0$ and where the integration is done along a straight line from 0 to $z$ in the $\xi$-plane. By integrating by part $m$ times, we obtain

$$
D_{z}^{\alpha} f(z)=\frac{d^{m}}{d z^{m}} D_{z}^{\alpha-m} f(z) .
$$

This allows modifying the restriction $\mathfrak{R}(\alpha)<0$ to $\Re(\alpha)<m$ [10].
In 1970, Osler [2] introduced a more general definition of the fractional derivative of a function $f(z)$ with respect to another function $g(z)$ based on Cauchy's integral formula.

Definition 1. Let $f(z)$ be analytic in the simply connected region $\mathscr{R}$. Let $g(z)$ be regular and univalent on $\mathscr{R}$ and let $g^{-1}(0)$ be an interior or boundary point of $\mathscr{R}$. Assume also that $\oint f(z) d z=0$ for any simple closed contour in $\mathscr{R} \cup\{0\}$ through $g^{-1}(0)$. Then if $\alpha$ is not a negative integer and $z$ is in $\mathscr{R}$, the fractional derivative of order $\alpha$ of $f(z)$ with respect to $g(z)$ is defined by

$$
D_{g(z)}^{\alpha} f(z)=\frac{\Gamma(\alpha+1)}{2 \pi i} \int_{g^{-1}(0)}^{(z+)} \frac{f(\zeta) g^{\prime}(\zeta)}{(g(\zeta)-g(z))^{\alpha+1}} d \zeta .
$$

For nonintegral $\alpha$, the integrand has a branch line which begins at $\zeta=z$ and passes through $\zeta=g^{-1}(0)$. The notation on this integral implies that the contour of integration starts at $g^{-1}(0)$, encloses $z$ once in the positive sense, and returns to $g^{-1}(0)$ without cutting the branch line.

With the use of that representation based on the Cauchy integral formula for the fractional derivatives, Osler gave a generalization of the following result [11, page 19] involving 
the derivative of order $N$ of the composite function $f(z)=$ $F(h(z))$ :

$$
D_{z}^{N} f(z)=\sum_{n=0}^{N} \frac{U_{n}(z) D_{h(z)}^{n} f(z)}{n !}
$$

where

$$
U_{n}(z)=\sum_{r=0}^{n}\left(\begin{array}{l}
n \\
r
\end{array}\right)(-h(z))^{r} D_{z}^{N} h(z)^{n-r}
$$

In particular, he found the following formula [4]:

$$
\begin{aligned}
D_{g(z)}^{\alpha} f(z) & =\sum_{n=-\infty}^{\infty}\left(\begin{array}{c}
\alpha \\
\gamma+n
\end{array}\right) D_{h(z)}^{\gamma+n} \frac{f(z)}{F(w, z)} \\
& \left.\cdot D_{h(z)}^{\alpha-\gamma-n}\left\{\frac{F(w, z) g^{\prime}(z)}{h^{\prime}(z)}\left(\frac{h(z)-h(w)}{g(z)-g(w)}\right)^{\alpha+1}\right\}\right|_{w=z},
\end{aligned}
$$

where the notation $D_{g(z)}^{\alpha} f(z)$ means the fractional derivative of order $\alpha$ of $f(z)$ with respect to $g(z)$. Osler proved the generalized chain rule by applying the generalized Leibniz rule [2] for fractional derivatives to an important fundamental relation involving fractional derivatives discovered also by Osler [4, page 290, Theorem 2]. The fundamental relation which is the central point of this paper is given by the next theorem.

Theorem 2. Let $f\left(g^{-1}(z)\right)$ and $f\left(h^{-1}(z)\right)$ be defined and analytic on the simply connected region $\mathscr{R}$ and let the origin be an interior or boundary point of $\mathscr{R}$. Suppose also that $g^{-1}(z)$ and $h^{-1}(z)$ are regular univalent functions on $\mathscr{R}$ and that $h^{-1}(0)=g^{-1}(0)$. Let $\oint f\left(g^{-1}(z)\right) d z$ vanish over simple closed contour in $\mathscr{R} \cup\{0\}$ through the origin. Then the following relation holds true:

$$
D_{g(z)}^{\alpha} f(z)=\left.D_{h(z)}^{\alpha}\left\{\frac{f(z) g^{\prime}(z)}{h^{\prime}(z)}\left(\frac{h(w)-h(z)}{g(w)-g(z)}\right)^{\alpha+1}\right\}\right|_{w=z}
$$

This fundamental relation is very useful to obtain very easily known and new summation formulas involving special functions of mathematical physics. For example, set $f(z)=$ $z^{p-2}, g(z)=z^{2}$, and $h(z)=z$ in (7). One sees easily that $g^{-1}(0)=h^{-1}(0)$. Thus, one has

$$
D_{z^{2}}^{\alpha} z^{p-2}=\left.D_{z}^{\alpha} 2 z^{p-1}(z+w)^{-\alpha-1}\right|_{w=z}
$$

The left-hand side is evaluated by using the well-known formula [12, page 83, Equation (2.4)]

$$
D_{z}^{\alpha} z^{q}=\frac{\Gamma(1+q)}{\Gamma(1+q-\alpha)} z^{q-\alpha} \quad(\Re(q)>-1)
$$

after replacing $z$ by $z^{2}$. Expanding $(w+z)^{-\alpha-1}$ in power series, using (9), and replacing $w$ by $z$ after operation, one obtains Kummer's summation formula

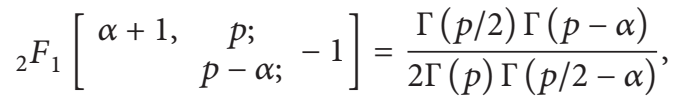

where

$$
{ }_{2} F_{1}\left[\begin{array}{rl}
a, & b ; \\
c ; & z
\end{array}\right]=\sum_{n=0}^{\infty} \frac{(a)_{n}(b)_{n}}{(c)_{n}} \frac{z^{n}}{n !}
$$

denotes the Gauss hypergeometric function [13] and $(\lambda)_{n}$ holds for the Pochhammer symbol defined, in terms of the Gamma function, by

$$
\begin{aligned}
(\lambda)_{n} & :=\frac{\Gamma(\lambda+n)}{\Gamma(\lambda)} \\
& = \begin{cases}\lambda(\lambda+1) \cdots(\lambda+n-1) & (n \in \mathbb{N} ; \lambda \in \mathbb{C}) \\
1 & (n=0 ; \lambda \in \mathbb{C} \backslash\{0\}) .\end{cases}
\end{aligned}
$$

In this paper, we present several new summation formulas involving special functions of mathematical physics obtained by using the fundamental relation (7). In Section 2, we introduce the Pochhammer based representation for fractional derivatives and we recall a well-poised fractional calculus operator given by Tremblay [14]. This well-poised operator will be used, throughout this paper, in order to ease the computations of fractional derivatives. Finally, Section 3 is devoted to the presentation of the main results. Many presumably new summation formulas are also given as special cases.

\section{Pochhammer Contour Integral Representation for Fractional Derivative and the Well-Poised Fractional Calculus Operator ${ }_{g(z)} O_{\beta}^{\alpha}$}

The less restrictive representation of fractional derivative according to parameters is Pochhammer's contour definition introduced in $[14,15]$.

Definition 3. Let $f(z)$ be analytic in a simply connected region $\mathscr{R}$. Let $g(z)$ be regular and univalent on $\mathscr{R}$ and let $g^{-1}(0)$ be an interior point of $\mathscr{R}$ then if $\alpha$ is not a negative integer, $p$ is not an integer, and $z$ is in $\mathscr{R}-\left\{g^{-1}(0)\right\}$, one defines the fractional derivative of order $\alpha$ of $g(z)^{p} f(z)$ with respect to $g(z)$ by

$$
\begin{aligned}
& D_{g(z)}^{\alpha} g(z)^{p} f(z) \\
& =\frac{\mathrm{e}^{-i \pi p} \Gamma(1+\alpha)}{4 \pi \sin (\pi p)} \\
& \quad \times \int_{C\left(z+, g^{-1}(0)+, z-, g^{-1}(0)-; F(a), F(a)\right)} \frac{f(\xi) g(\xi)^{p} g^{\prime}(\xi)}{(g(\xi)-g(z))^{\alpha+1}} d \xi .
\end{aligned}
$$




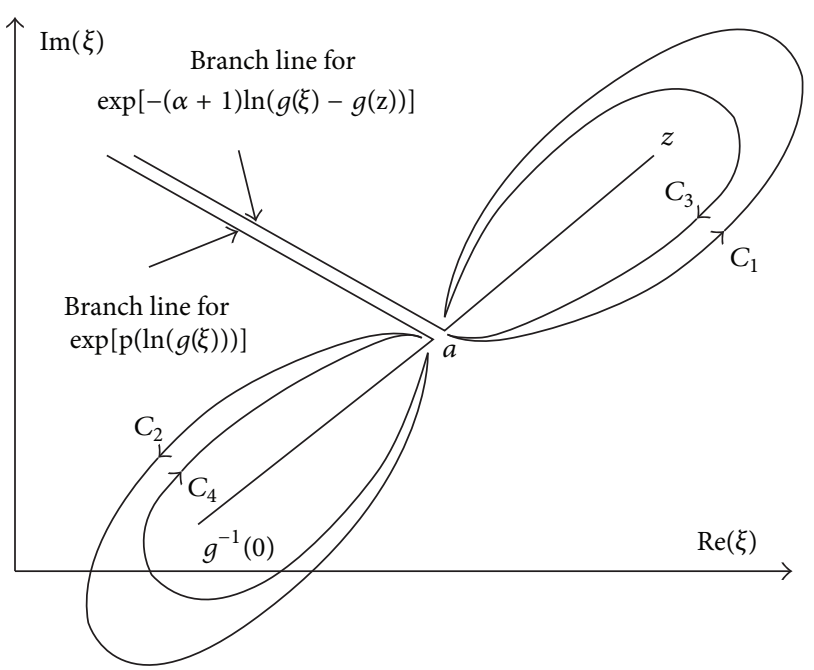

FIgURE 1: Pochhammer's contour.

For nonintegers $\alpha$ and $p$, the functions $g(\xi)^{p}$ and $(g(\xi)-$ $g(z))^{-\alpha-1}$ in the integrand have two branch lines which begin, respectively, at $\xi=z$ and $\xi=g^{-1}(0)$, and both pass through the point $\xi=a$ without crossing the Pochhammer contour $P(a)=\left\{C_{1} \cup C_{2} \cup C_{3} \cup C_{4}\right\}$ at any other point as shown in Figure 1. $F(a)$ denotes the principal value of the integrand in (13) at the beginning and ending point of the Pochhammer contour $P(a)$ which is closed on Riemann surface of the multiple-valued function $F(\xi)$.

Remark 4. In Definition 3, the function $f(z)$ must be analytic at $\xi=g^{-1}(0)$. However it is interesting to note here that we could also allow $f(z)$ to have an essential singularity at $\xi=$ $g^{-1}(0)$, and (13) would still be valid.

Remark 5. The Pochhammer contour never crosses the singularities at $\xi=g^{-1}(0)$ and $\xi=z$ in (13); then we know that the integral is analytic for all $p$ and for all $\alpha$ and for $z$ in $\mathscr{R}-\left\{g^{-1}(0)\right\}$. Indeed, the only possible singularities of $D_{g(z)}^{\alpha} g(z)^{p} f(z)$ are $\alpha=-1,-2, \ldots$, and $p=0, \pm 1, \pm 2, \ldots$ which can directly be identified from the coefficient of the integral (13). However, integrating by parts $N$ times the integral in (13) by two different ways, we can show that $\alpha=$ $-1,-2, \ldots$, and $p=0,1,2, \ldots$ are removable singularities (see $[15])$.

Remark 6. A deep and complete study of the fundamental properties of the function $D_{z}^{\alpha} z^{p}(\ln z)^{\delta} f(z)$ with $\delta=0$ or 1 , relative to the the values of the parameters $z$, $p$, and $\alpha$, can be found in [16].

In 1974, Tremblay [14] introduced the operator ${ }_{g(z)} O_{\beta}^{\alpha}$ in order to deal with special functions more efficiently and to facilitate the obtention of new relations such as hypergeometric transformations. This operator will be useful in the proofs of the summation formulas given in the next section.
The operator ${ }_{g(z)} \mathrm{O}_{\beta}^{\alpha}$ is defined in terms of the fractional calculus operator $D_{g(z)}^{\alpha}$ as

$$
{ }_{g(z)} O_{\beta}^{\alpha}:=\frac{\Gamma(\beta)}{\Gamma(\alpha)} g(z)^{1-\beta} D_{g(z)}^{\alpha-\beta} g(z)^{\alpha-1}
$$

with $\beta \neq 0,-1,-2, \ldots$

This operator has been used very recently in [3] to prove a new generalized Leibniz rule for fractional derivatives as well as in [17] to obtain some new series involving special functions.

This operator has many very useful properties. We chose to give only one of them which will be used in the proofs of the main results; that is,

$$
\begin{aligned}
g(z) & O_{\beta}^{\alpha} g(z)^{\lambda} f(z) \\
& =\frac{\Gamma(\beta) \Gamma(\alpha+\lambda)}{\Gamma(\alpha) \Gamma(\beta+\lambda)} g(z)^{\lambda}{ }_{g(z)} O_{\beta+\lambda}^{\alpha+\lambda} f(z) .
\end{aligned}
$$

In terms of the fractional calculus operator ${ }_{z} \mathrm{O}_{\beta}^{\alpha}$, the modified fundamental relation (7) holds the following form:

$$
\begin{aligned}
g(z) & O_{\beta}^{\alpha} f(z) \\
= & \left(\frac{g(z)}{h(z)}\right)^{1-\beta} h(z) O_{\beta}^{\alpha} \\
& \times\left.\left\{f(z)\left(\frac{g(z)}{h(z)}\right)^{\alpha-1} \frac{g^{\prime}(z)}{h^{\prime}(z)}\left(\frac{g(w)-g(z)}{h(w)-h(z)}\right)^{\beta-\alpha-1}\right\}\right|_{w=z}
\end{aligned}
$$

with $\beta \neq 0,-1,-2, \ldots$

It is worthy to mention that the operator ${ }_{g(z)} \mathrm{O}_{\beta}^{\alpha}$ has a lot more interesting properties and applications.

\section{Main Results and Special Cases}

In this section, we present a new general formula related to the generalized chain rule. We give many special cases involving special functions such as the first Appell function $F_{1}$, the Lauricella function of several variables $F_{D}^{(n)}$, and the generalized hypergeometric functions. These functions are evaluated most of the time at arguments related to the roots of unity.

Main Formula. Consider

$$
\begin{aligned}
& z_{z^{p}} O_{\beta}^{\alpha} f(z) \\
& =\frac{p \Gamma(\beta) \Gamma(p \alpha / q)}{q \Gamma(\alpha) \Gamma(\beta+(p-q) \alpha / q)} \\
& \quad \times{ }_{z^{q} O_{\beta+(p-q) \alpha / q} \alpha+(p-q) \alpha / q}\left\{f(z) \prod_{s=1}^{p-1}\left(1-\frac{z}{w} \mathrm{e}^{-2 \pi i s / p}\right)^{\beta-\alpha-1}\right. \\
& \left.\quad \times \prod_{r=1}^{q-1}\left(1-\frac{z}{w} \mathrm{e}^{-2 \pi i r / q}\right)^{1+\alpha-\beta}\right\}\left.\right|_{w=z}
\end{aligned}
$$

with $\beta \neq 0,-1,-2, \ldots$ and $\beta-(p-q) \alpha / q \neq 0,-1,-2, \ldots$. 
Proof. Let $g(z)=z^{p}$ and let $h(z)=z^{q}$ with $p$ and $q$ two positive integers in (16). We have

$$
\begin{aligned}
z^{p} O_{\beta}^{\alpha} & f(z) \\
= & \left(z^{p-q}\right)^{1-\beta} z^{q} O_{\beta}^{\alpha} \\
& \times\left.\left\{f(z)\left(z^{p-q}\right)^{\alpha} \frac{p}{q}\left(\frac{z^{p}-w^{p}}{z^{q}-w^{q}}\right)^{\beta-\alpha-1}\right\}\right|_{w=z} \\
= & \frac{p}{q}\left(z^{p-q}\right)^{1-\beta} z^{q} O_{\beta}^{\alpha} \\
& \times\left.\left\{f(z)\left(z^{q}\right)^{(p-q) \alpha / q}\left(\frac{z^{p}-w^{p}}{z^{q}-w^{q}}\right)^{\beta-\alpha-1}\right\}\right|_{w=z} \\
= & \frac{p}{q}\left(z^{p-q}\right)^{-\alpha} z^{q} O_{\beta}^{\alpha} \\
& \times\left\{f(z)\left(z^{q}\right)^{(p-q) \alpha / q}\left(1-\left(\frac{z}{w}\right)^{p}\right)^{\beta-\alpha-1}\right. \\
& \left.\quad \times\left(1-\left(\frac{z}{w}\right)^{q}\right)^{1+\alpha-\beta}\right\} \mid
\end{aligned}
$$

With the help of (15), the last equation becomes

$$
\begin{aligned}
z^{p} O_{\beta}^{\alpha} f(z) \\
=\frac{p \Gamma(\beta) \Gamma(p \alpha / q)}{q \Gamma(\alpha) \Gamma(\beta+(p-q) \alpha / q)} z^{q} O_{\beta+(p-q) \alpha / q}^{\alpha+(p-q) \alpha / q} \\
\quad \times\left.\left\{f(z)\left(1-\left(\frac{z}{w}\right)^{p}\right)^{\beta-\alpha-1}\left(1-\left(\frac{z}{w}\right)^{q}\right)^{1+\alpha-\beta}\right\}\right|_{w=z} .
\end{aligned}
$$

Using the fact that both $p$ and $q$ are positive integers, the result follows.

Example 7. Let $f(z)=1, p=2$, and $q=4$ in (17). We, thus, have

$$
\begin{aligned}
1= & \frac{\Gamma(\beta) \Gamma(\alpha / 2)}{2 \Gamma(\alpha) \Gamma(\beta-\alpha / 2)} z^{4} O_{\beta-\alpha / 2}^{\alpha / 2} \\
& \times\left.\left\{\left(1+\frac{z}{w}\right)^{\beta-\alpha-1} \prod_{r=1}^{3}\left(1-\frac{z}{w} \mathrm{e}^{-2 \pi i r / 3}\right)^{1+\alpha-\beta}\right\}\right|_{w=z} \\
= & \frac{\Gamma(\beta) \Gamma(\alpha / 2)}{2 \Gamma(\alpha) \Gamma(\beta-\alpha / 2)} z^{4} O_{\beta-\alpha / 2}^{\alpha / 2} \\
& \times\left.\left\{\left(1-i \frac{z}{w}\right)^{1+\alpha-\beta}\left(1+i \frac{z}{w}\right)^{1+\alpha-\beta}\right\}\right|_{w=z} \\
= & \left.\frac{\Gamma(\beta) \Gamma(\alpha / 2)}{2 \Gamma(\alpha) \Gamma(\beta-\alpha / 2)} z^{4} O_{\beta-\alpha / 2}^{\alpha / 2}\left\{\left(1+\frac{z^{2}}{w^{2}}\right)^{1+\alpha-\beta}\right\}\right|_{w=z} .
\end{aligned}
$$

Expanding $\left(1+z^{2} / w^{2}\right)^{1+\alpha-\beta}$ in power series, (20) becomes

$$
\begin{aligned}
& 2 \Gamma(\alpha) \Gamma(\beta-\alpha / 2) \\
& \Gamma(\beta) \Gamma(\alpha / 2) \\
& =\sum_{n=0}^{\infty} \frac{(\beta-\alpha-1)_{n}(-1)^{n}}{n ! z^{2 n}} z^{4} O_{\beta-\alpha / 2}^{\alpha / 2}\left(z^{4}\right)^{n / 2} \\
& =\sum_{n=0}^{\infty} \frac{(\beta-\alpha-1)_{n}(-1)^{n}}{n !} \frac{\Gamma(\beta-\alpha / 2) \Gamma((\alpha+n) / 2)}{\Gamma(\alpha / 2) \Gamma(\beta-(\alpha-n) / 2)} .
\end{aligned}
$$

The right-hand side of (21) can be split in two parts

$$
\begin{aligned}
\sum_{n=0}^{\infty} & \frac{(\beta-\alpha-1)_{n}(-1)^{n}}{n !} \frac{\Gamma(\beta-\alpha / 2) \Gamma((\alpha+n) / 2)}{\Gamma(\alpha / 2) \Gamma(\beta-(\alpha-n) / 2)} \\
= & \sum_{n=0}^{\infty} \frac{(\beta-\alpha-1)_{2 n}}{(2 n) !} \frac{\Gamma(\beta-\alpha / 2) \Gamma(\alpha / 2+n)}{\Gamma(\alpha / 2) \Gamma(\beta-\alpha / 2+n)} \\
& -\sum_{n=0}^{\infty} \frac{(\beta-\alpha-1)_{2 n+1}}{(2 n+1) !} \frac{\Gamma(\beta-\alpha / 2) \Gamma((\alpha+1) / 2+n)}{\Gamma(\alpha / 2) \Gamma(\beta-(\alpha-1) / 2+n)} .
\end{aligned}
$$

Converting the terms involving Gamma function in the last expression into Pochhammer's symbol and making some simplifications, we find

$$
\begin{aligned}
\sum_{n=0}^{\infty} & \frac{(\beta-\alpha-1)_{n}(-1)^{n}}{n !} \frac{\Gamma(\beta-\alpha / 2) \Gamma((\alpha+n) / 2)}{\Gamma(\alpha / 2) \Gamma(\beta-(\alpha-n) / 2)} \\
= & \sum_{n=0}^{\infty} \frac{((\beta-\alpha-1) / 2)_{n}((\beta-\alpha) / 2)_{n}(\alpha / 2)_{n}}{(\beta-\alpha / 2)_{n}(1 / 2)_{n} n !} \\
& -\frac{(\beta-\alpha-1) \Gamma(\beta-\alpha / 2) \Gamma((\alpha+1) / 2)}{\Gamma(\alpha / 2) \Gamma(\beta-(\alpha-1) / 2)} \\
& \times \sum_{n=0}^{\infty} \frac{((\beta-\alpha+1) / 2)_{n}((\beta-\alpha) / 2)_{n}((\alpha+1) / 2)_{n}}{(\beta-(\alpha-1) / 2)_{n}(3 / 2)_{n} n !} .
\end{aligned}
$$

Finally, rewriting the right-hand side of (23) in terms of generalized hypergeometric function and combining with (21), we obtain the following summation formula:

$$
\begin{aligned}
& \frac{2 \Gamma(\alpha) \Gamma(\beta-\alpha / 2)}{\Gamma(\beta) \Gamma(\alpha / 2)} \\
& ={ }_{3} F_{2}\left[\begin{array}{c}
\frac{\beta-\alpha-1}{2}, \frac{\beta-\alpha}{2}, \frac{\alpha}{2} ; \\
\beta-\frac{\alpha}{2}, \frac{1}{2} ;
\end{array}\right] \\
& -\frac{(\beta-\alpha-1) \Gamma(\beta-\alpha / 2) \Gamma((\alpha+1) / 2)}{\Gamma(\alpha / 2) \Gamma(\beta-(\alpha-1) / 2)} \\
& \times{ }_{3} F_{2}\left[\frac{\beta-\alpha}{2}, \frac{\beta-\alpha+1}{2}, \frac{\alpha+1}{2} ; \quad \frac{\alpha-1}{2}, \frac{3}{2} ;\right] .
\end{aligned}
$$


Special Cases with $q=1$. Consider

$$
\begin{aligned}
{ }_{z^{p}} O_{\beta}^{\alpha} f(z) & \\
= & p\left(z^{p-1}\right)^{-\alpha}{ }_{z} O_{\beta}^{\alpha} \\
& \times\left.\left\{f(z)\left(z^{p-1}\right)^{\alpha} \prod_{s=1}^{p-1}\left(1-\frac{z}{w} \mathrm{e}^{-2 \pi i s / p}\right)^{\beta-\alpha-1}\right\}\right|_{w=z} .
\end{aligned}
$$

Example 8. Setting $f(z)=1$ and $p=3$ in (25) gives

$$
\begin{aligned}
1= & 3 z^{-2 \alpha} O_{\beta}^{\alpha} \\
& \times\left.\left\{z^{2 \alpha}\left(1-\frac{z}{w} \mathrm{e}^{-2 \pi i / 3}\right)^{\beta-\alpha-1}\left(1-\frac{z}{w} \mathrm{e}^{-4 \pi i / 3}\right)^{\beta-\alpha-1}\right\}\right|_{w=z} \\
= & \frac{3 \Gamma(\beta) \Gamma(3 \alpha)}{\Gamma(\alpha) \Gamma(\beta+2 \alpha)} z O_{\beta+2 \alpha}^{3 \alpha} \\
& \times\left.\left\{\left(1-\frac{z}{w} \mathrm{e}^{-2 \pi i / 3}\right)^{\beta-\alpha-1}\left(1-\frac{z}{w} \mathrm{e}^{-4 \pi i / 3}\right)^{\beta-\alpha-1}\right\}\right|_{w=z} .
\end{aligned}
$$

Observe that

$$
\begin{aligned}
{ }_{z} O_{b}^{a}(1-\gamma z)^{-\mu}(1-\lambda z)^{-\nu} \\
\quad=\sum_{i=0}^{\infty} \sum_{j=0}^{\infty} \frac{(a)_{i+j}(\mu)_{i}(\nu)_{j}}{(b)_{i+j}} \frac{(\gamma z)^{i}}{i !} \frac{(\lambda z)^{j}}{j !} \\
\quad=F_{1}(a, \mu, \nu ; b ; \gamma z, \lambda z),
\end{aligned}
$$

where $F_{1}(a, \mu, v ; b ; \gamma z, \lambda z)$ denotes the first Appell function [18]. Replacing (27) in (26) yields the following summation formula:

$$
\begin{aligned}
F_{1} & \left(3 \alpha, 1+\alpha-\beta, 1+\alpha-\beta ; \beta+2 \alpha ; \mathrm{e}^{-2 \pi i / 3}, \mathrm{e}^{-4 \pi i / 3}\right) \\
& =\frac{\Gamma(\alpha) \Gamma(\beta+2 \alpha)}{3 \Gamma(\beta) \Gamma(3 \alpha)} .
\end{aligned}
$$

It is interesting to note that setting $\beta=2 / 3$ and replacing $\alpha$ by $2 \alpha$ in (28), and using the well-known identity [19],

$$
\begin{aligned}
F_{1} & \left(a, b, b^{\prime} ; b+b^{\prime} ; x, y\right) \\
& =(1-y)^{-a}{ }_{2} F_{1}\left[\begin{array}{cc}
a, & b ; \\
b+b^{\prime} ; & \frac{x-y}{1-y}
\end{array}\right]
\end{aligned}
$$

reduces to the known summation formula [20]

$$
\begin{aligned}
& { }_{2} F_{1}\left[\begin{array}{cc}
6 \alpha, & \frac{1}{3}+\alpha ; \\
& \frac{2}{3}+4 \alpha ;
\end{array} \mathrm{e}^{-\pi i / 3}\right] \\
& =3^{-3 \alpha} \mathrm{e}^{-\alpha \pi i} \frac{\Gamma(2 / 3+4 \alpha) \Gamma(1 / 3)}{\Gamma(1 / 3+2 \alpha) \Gamma(2 / 3+2 \alpha)} \text {. }
\end{aligned}
$$

Furthermore, with the help of the following reduction formulas for the Appell $F_{1}$ functions given by Nagel [21],

$$
\begin{aligned}
& F_{1}\left(a, b, b ; \gamma ; \frac{1}{x}, \frac{1}{y}\right) \\
& =\left(\frac{x y}{(x-1)(y-1)}\right)^{b} \\
& \times \sum_{n=0}^{\infty} \frac{(\gamma-a)_{n}(b)_{n}}{(\gamma)_{n} n !}\left(\frac{1-x-y}{(x-1)(y-1)}\right)^{n} \\
& \times{ }_{2} F_{1}\left[\begin{array}{cc}
-n, & a ; \\
& \gamma+n ; \frac{1}{x+y-1}
\end{array}\right], \\
& F_{1}(a, b, b ; \gamma ; x, y) \\
& =(1-x-y)^{-b} \\
& \times \sum_{n=0}^{\infty} \frac{(\gamma-a)_{n}(b)_{n}}{(\gamma)_{n} n !}\left(\frac{x+y}{x+y-1}\right)^{n} \\
& \times{ }_{3} F_{2}\left[\begin{array}{ccc}
\frac{a}{2}, & \frac{a+1}{2}, & b+n ; \\
\frac{\gamma+n}{2}, & \frac{\gamma+n+1}{2} ; & \frac{x y}{x+y-1}
\end{array}\right]
\end{aligned}
$$

in conjunction with (28) give, respectively, after simplifications the two summation formulas

$$
\begin{aligned}
& \sum_{n=0}^{\infty} \frac{(\beta-\alpha)_{n}(1+\alpha-\beta)_{n}}{(\beta+2 \alpha)_{n} n !}\left(\frac{2}{3}\right)^{n} \\
& \times{ }_{2} F_{1}\left[\begin{array}{cc}
-n, & 3 \alpha ; \\
& \beta+2 \alpha+n ;
\end{array}\right] \\
& =\frac{\Gamma(\alpha) \Gamma(\beta+2 \alpha)}{3^{\beta-\alpha} \Gamma(\beta) \Gamma(3 \alpha)}, \\
& \sum_{n=0}^{\infty} \frac{(\beta-\alpha)_{n}(1+\alpha-\beta)_{n}}{(\beta+2 \alpha)_{n} n !}\left(\frac{1}{2}\right)^{n} \\
& \times{ }_{3} F_{2}\left[\begin{array}{ccc}
\frac{3 \alpha}{2}, & \frac{3 \alpha+1}{2}, & 1+\alpha-\beta+n ; \\
\frac{2 \alpha+\beta+n}{2}, & \frac{2 \alpha+\beta+n+1}{2} ; & -\frac{1}{2}
\end{array}\right] \\
& =\frac{2^{1-\alpha-\beta} \Gamma(\alpha) \Gamma(\beta+2 \alpha)}{3 \Gamma(\beta) \Gamma(3 \alpha)} \text {. }
\end{aligned}
$$

Example 9. Let $f(z)=1$ and let $p$ be an integer in (25). We have that

$$
\begin{aligned}
1= & p\left(z^{p-1}\right)^{-\alpha} O_{\beta}^{\alpha} \\
& \times\left.\left\{\left(z^{p-1}\right)^{\alpha} \prod_{s=1}^{p-1}\left(1-\frac{z}{w} \mathrm{e}^{-2 \pi i s / p}\right)^{\beta-\alpha-1}\right\}\right|_{w=z}
\end{aligned}
$$




$$
\begin{aligned}
= & p \frac{\Gamma(\beta) \Gamma(p \alpha)}{\Gamma(\alpha) \Gamma(\beta+(p-1) \alpha)}{ }_{z} O_{\beta+(p-1) \alpha}^{p \alpha} \\
& \times\left.\left\{\prod_{s=1}^{p-1}\left(1-\frac{z}{w} \mathrm{e}^{-2 \pi i s / p}\right)^{\beta-\alpha-1}\right\}\right|_{w=z} .
\end{aligned}
$$

The Lauricella function $F_{D}^{(n)}$ of $n$ variables is defined as [18, page 60]

$$
\begin{gathered}
F_{D}^{(n)}\left[a, b_{1}, \ldots, b_{n} ; c ; x_{1}, \ldots, x_{n}\right] \\
=\sum_{m_{1}, \ldots, m_{n}=0}^{\infty} \frac{(a)_{m_{1}+\cdots+m_{n}}\left(b_{1}\right)_{m_{1}} \cdots\left(b_{n}\right)_{m_{n}}}{(c)_{m_{1}+\cdots+m_{n}}} \\
\times \frac{x_{1}^{m_{1}}}{m_{1} !} \cdots \frac{x_{n}^{m_{n}}}{m_{n} !}
\end{gathered}
$$

with $\max \left\{\left|x_{1}\right|, \ldots,\left|x_{n}\right|\right\}<1$. We can easily see that the following relation,

$$
\begin{aligned}
F_{D}^{(n)} & {\left[a, \gamma_{1}, \ldots, \gamma_{n} ; b ; c_{1} z, \ldots, c_{n} z\right] } \\
= & { }_{z} O_{b}^{a} \prod_{k=1}^{n}\left(1-c_{k} z\right)^{-\gamma_{k}},
\end{aligned}
$$

holds true. So, using this last relation in conjunction with (33) provides the following summation formula:

$$
\begin{gathered}
F_{D}^{(p-1)}[p \alpha, 1+\alpha-\beta, \ldots, 1+\alpha-\beta ; \beta+(p-1) \alpha ; \\
\left.\quad \mathrm{e}^{-2 \pi i / p}, \mathrm{e}^{-4 \pi i / p}, \ldots, \mathrm{e}^{-2(p-1) \pi i / p}\right] \\
=\frac{\Gamma(\alpha) \Gamma(\beta+(p-1) \alpha)}{p \Gamma(\beta) \Gamma(p \alpha)} .
\end{gathered}
$$

Let us examine another special case of (17) when $f(z)$ is not equal to 1 .

Example 10. Set $p=3$ and $f(z)=\mathrm{e}^{z^{3}}$ in (25). We have

$$
\begin{aligned}
z^{3} O_{\beta}^{\alpha} \mathrm{e}^{z^{3}}= & 3 z^{-2 \alpha} \mathrm{O}_{\beta}^{\alpha} \\
& \times\left.\left\{\mathrm{e}^{z^{3}} z^{2 \alpha} \prod_{s=1}^{2}\left(1-\frac{z}{w} \mathrm{e}^{-2 \pi i s / 3}\right)^{\beta-\alpha-1}\right\}\right|_{w=z} .
\end{aligned}
$$

Expanding $\mathrm{e}^{z^{3}}$ in power series, we find for the left-hand side of (37)

$$
z^{3} O_{\beta}^{\alpha} e^{z^{3}}={ }_{1} F_{1}\left[\begin{array}{l}
\alpha ; \\
\beta ;
\end{array} z^{3}\right]
$$

The right-hand side of (37) yields after expanding $\mathrm{e}^{z^{3}}$ in power series and using property (15)

$$
\begin{aligned}
& \frac{3 \Gamma(\beta)}{\Gamma(\alpha)} \sum_{n=0}^{\infty} \frac{\Gamma(3 \alpha+3 n)}{\Gamma(2 \alpha+\beta+3 n)} \frac{z^{3 n}}{n !} \\
& \quad \times\left.{ }_{z} O_{2 \alpha+\beta+3 n}^{3 \alpha+3 n} \prod_{s=1}^{2}\left(1-\frac{z}{w} \mathrm{e}^{-2 \pi i s / 3}\right)^{\beta-\alpha-1}\right|_{w=z} .
\end{aligned}
$$

Using (27) and making some elementary simplifications, the last formula reduces to

$$
\begin{array}{r}
\frac{3 \Gamma(\beta) \Gamma(3 \alpha)}{\Gamma(\alpha) \Gamma(2 \alpha+\beta)} \sum_{n=0}^{\infty} \frac{(3 \alpha)_{3 n}}{(2 \alpha+\beta)_{3 n}} \frac{z^{3 n}}{n !} \\
\times F_{1}(3 \alpha+3 n, 1+\alpha-\beta, 1+\alpha-\beta ; \\
\left.\beta+2 \alpha+3 n ; \mathrm{e}^{-2 \pi i / 3}, \mathrm{e}^{-4 \pi i / 3}\right) .
\end{array}
$$

Combining (38) and (40) and putting $z=1$ provide the following presumably new summation formula:

$$
\begin{aligned}
& \sum_{n=0}^{\infty} \frac{(3 \alpha)_{3 n}}{(2 \alpha+\beta)_{3 n} n !} \\
& \quad \times F_{1}(3 \alpha+3 n, 1+\alpha-\beta, 1+\alpha-\beta ; \\
& \left.\beta+2 \alpha+3 n ; \mathrm{e}^{-2 \pi i / 3}, \mathrm{e}^{-4 \pi i / 3}\right) \\
& =\frac{\Gamma(\alpha) \Gamma(2 \alpha+\beta)}{3 \Gamma(\beta) \Gamma(3 \alpha)}{ }_{1} F_{1}\left[\begin{array}{c}
\alpha ; \\
\beta ;
\end{array}\right] .
\end{aligned}
$$

Cases with $p=1$. Consider

$$
\begin{aligned}
& \frac{q \Gamma(\alpha) \Gamma(\beta-\alpha+\alpha / q)}{\Gamma(\beta) \Gamma(\alpha / q)}{ }_{z} O_{\beta}^{\alpha} f(z) \\
& ={ }_{z^{q}} O_{\beta-\alpha+\alpha / q}^{\alpha / q} f(z) \\
& \quad \times\left.\prod_{s=1}^{q-1}\left(1-\frac{z}{w} \mathrm{e}^{-2 \pi i s / q}\right)^{1+\alpha-\beta}\right|_{w=z} .
\end{aligned}
$$

Example 11. Let $f(z)=1$ and $q=3$ in (42). We obtain

$$
\begin{aligned}
& \frac{3 \Gamma(\alpha) \Gamma(\beta-2 \alpha / 3)}{\Gamma(\beta) \Gamma(\alpha / 3)} \\
& =z^{3} O_{\beta-2 \alpha / 3}^{\alpha / 3} \\
& \quad \times\left.\left\{\left(1-\frac{z}{w} e^{-2 \pi i / 3}\right)^{1+\alpha-\beta}\left(1-\frac{z}{w} e^{-4 \pi i / 3}\right)^{1+\alpha-\beta}\right\}\right|_{w=z} .
\end{aligned}
$$

Expanding in power series, we find for the right-hand side of (43)

$$
\begin{aligned}
\left.z^{3} O_{\beta-2 \alpha / 3}^{\alpha / 3}\left\{\left(1-\frac{z}{w} \mathrm{e}^{-2 \pi i / 3}\right)^{1+\alpha-\beta}\left(1-\frac{z}{w} \mathrm{e}^{-4 \pi i / 3}\right)^{1+\alpha-\beta}\right\}\right|_{w=z} \\
=\sum_{k=0}^{\infty} \sum_{j=0}^{\infty} \frac{(\beta-\alpha-1)_{k} \mathrm{e}^{-2 \pi i k / 3}}{k !} \\
\times \frac{(\beta-\alpha-1)_{j} \mathrm{e}^{-4 \pi i j / 3}}{j !} \frac{1}{z^{k+j}} \\
\times{ }_{z^{3} O_{\beta-2 \alpha / 3}^{\alpha / 3}\left(z^{3}\right)^{(k+j) / 3}}
\end{aligned}
$$




$$
\begin{aligned}
&=\sum_{k=0}^{\infty} \sum_{j=0}^{\infty} \frac{(\beta-\alpha-1)_{k} \mathrm{e}^{-2 \pi i k / 3}}{k !} \\
& \times \frac{(\beta-\alpha-1)_{j} \mathrm{e}^{-4 \pi i j / 3}}{j !} \\
& \times \frac{\Gamma(\beta-2 \alpha / 3) \Gamma((\alpha+k+j) / 3)}{\Gamma(\beta-(2 \alpha-k-j) / 3) \Gamma(\alpha / 3)} \\
& \sum_{k=0}^{\infty} \frac{(\beta-\alpha-1)_{k} \mathrm{e}^{-2 \pi i k / 3}}{k !} \\
& \times \frac{\Gamma(\beta-2 \alpha / 3) \Gamma((\alpha+k) / 3)}{\Gamma(\beta-(2 \alpha-k) / 3) \Gamma(\alpha / 3)} \\
& \times{ }_{2} F_{1}\left[\begin{array}{c}
-k, \quad \beta-\alpha-1 ; \\
2+\alpha-\beta-k ;
\end{array}\right] .
\end{aligned}
$$

We, thus, get the following (presumably) new summation formula:

$$
\begin{aligned}
& \sum_{k=0}^{\infty} \frac{(\beta-\alpha-1)_{k} \mathrm{e}^{-2 \pi i k / 3}}{k !} \frac{\Gamma((\alpha+k) / 3)}{\Gamma(\beta-(2 \alpha-k) / 3)} \\
& \quad \times{ }_{2} F_{1}\left[\begin{array}{cc}
-k, \quad \beta-\alpha-1 ; & \mathrm{e}^{-2 \pi i / 3} \\
2+\alpha-\beta-k ;
\end{array}\right] \\
& =\frac{3 \Gamma(\alpha)}{\Gamma(\beta)} .
\end{aligned}
$$

Remark 12. The cases where $\beta=\alpha-n$ with $n=0,1,2, \ldots$ must be treated very carefully as $\lim _{\beta \rightarrow \alpha-n}$.

\section{Conflict of Interests}

The authors declare that there is no conflict of interests regarding the publication of this paper.

\section{References}

[1] L. M. B. Campos, "On a concept of derivative of complex order with applications to special functions," IMA Journal of Applied Mathematics, vol. 33, no. 2, pp. 109-133, 1984.

[2] T. J. Osler, "Leibniz rule for fractional derivatives generalized and an application to infinite series," SIAM Journal on Applied Mathematics, vol. 18, pp. 658-674, 1970.

[3] R. Tremblay, S. Gaboury, and B. Fugère, "A new Leibniz rule and its integral analogue for fractional derivatives," Integral Transforms and Special Functions, vol. 24, no. 2, pp. 111-128, 2013.

[4] T. J. Osler, "The fractional derivative of a composite function," SIAM Journal on Mathematical Analysis, vol. 1, pp. 288-293, 1970.

[5] T. J. Osler, "Taylor's series generalized for fractional derivatives and applications," SIAM Journal on Mathematical Analysis, vol. 2, pp. 37-48, 1971.
[6] R. Tremblay and B. Fugère, "The use of fractional derivatives to expand analytical functions in terms of quadratic functions with applications to special functions," Applied Mathematics and Computation, vol. 187, no. 1, pp. 507-529, 2007.

[7] R. Tremblay, S. Gaboury, and B. Fugère, "Taylor-like expansion in terms of a rational function obtained by means of fractional derivatives," Integral Transforms and Special Functions, vol. 24, no. 1, pp. 50-64, 2013.

[8] R. Tremblay, S. Gaboury, and B. Fugère, "A new transformation formula for fractional derivatives with applications," Integral Transforms and Special Functions, vol. 24, no. 3, pp. 172-186, 2013.

[9] J. Liouville, "Memoire sur le calcul des differentielles a indices quelconques," Journal de l'École Polytechnique, vol. 13, pp. 71$162,1832$.

[10] M. Riesz, "Lintegrale de Riemann-Liouville et le probleme de Cauchy," Acta Mathematica, vol. 81, pp. 1-233, 1949.

[11] I. S. Gradshteyn and I. M. Ryzhkl, Table of Integrals, Series and Products, Academic Press, New York, NY, USA, 1965.

[12] K. S. Miller and B. Ross, An Introduction of the Fractional Calculus and Fractional Differential Equations, John Wiley \& Sons, New York, NY, USA, 1993.

[13] E. D. Rainville, Special Functions, Macmillan Company, New York, NY, USA, 1960.

[14] R. Tremblay, Une contribution a la theorie de la derivee fractionnaire [Ph.D. thesis], Laval University, Quebec City, Canada, 1974.

[15] J.-L. Lavoie, T. J. Osler, and R. Tremblay, "Fundamental properties of fractional derivatives via pochhammer integrals," in Fractional Calculus and Its Applications, vol. 457 of Lecture Notes in Mathematics, pp. 323-356, Springer, 1975.

[16] J.-L. Lavoie, T. J. Osler, and R. Tremblay, "Fundamental properties of fractional derivatives via pochhammer integrals," in Fractional Calculus and Its Applications, vol. 457 of Lecture Notes in Mathematics, pp. 323-356, Springer, Berlin, Germany, 1975.

[17] S. Gaboury and R. Tremblay, "A note on some new series of special functions," Integral Transforms and Special Functions, vol. 25, no. 5, pp. 336-343, 2014.

[18] H. M. Srivastava and P. W. Karlsson, Multiple Gaussian Hypergeometric Series, Halsted Press (Ellis Horwoor Limited), John Wiley and Sons, Chichester, UK, 1985.

[19] L. J. Slater, Generalized Hypergeometric Functions, Cambridge University Press, Cambridge, UK, 1966.

[20] A. Erdelyi, W. Magnus, F. Oberhettinger, and F. Tricomi, Higher Transcendental Functions, vol. 1-3, McGraw-Hill, New York, NY, USA, 1953.

[21] B. Nagel, "Some integrals and expansions related to the hypergeometric functions $F_{1}$ and $\Phi_{1}$ in two variables," Arkiv för Fysik, vol. 24, pp. 479-493, 1963. 


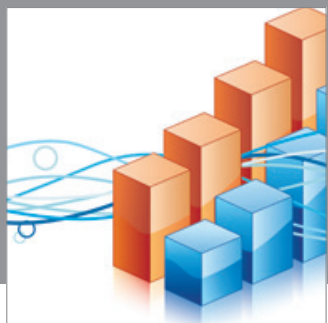

Advances in

Operations Research

mansans

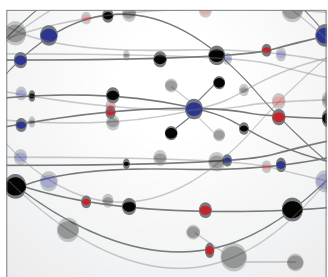

The Scientific World Journal
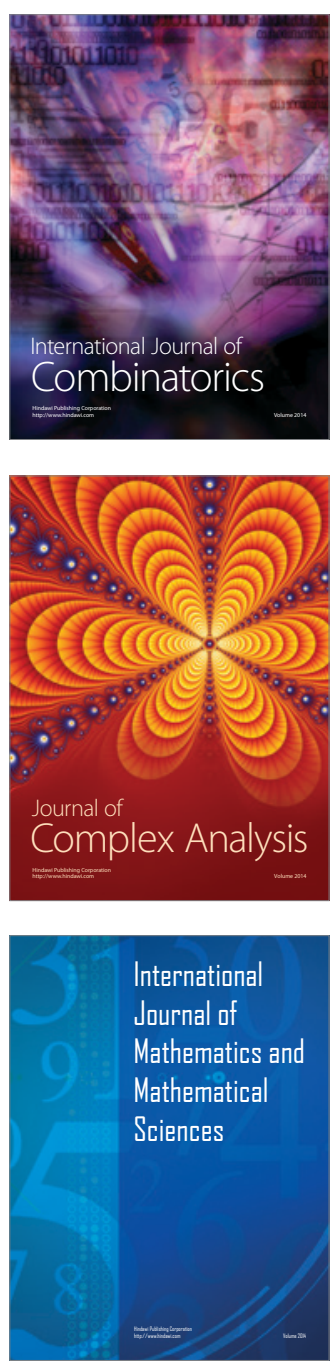
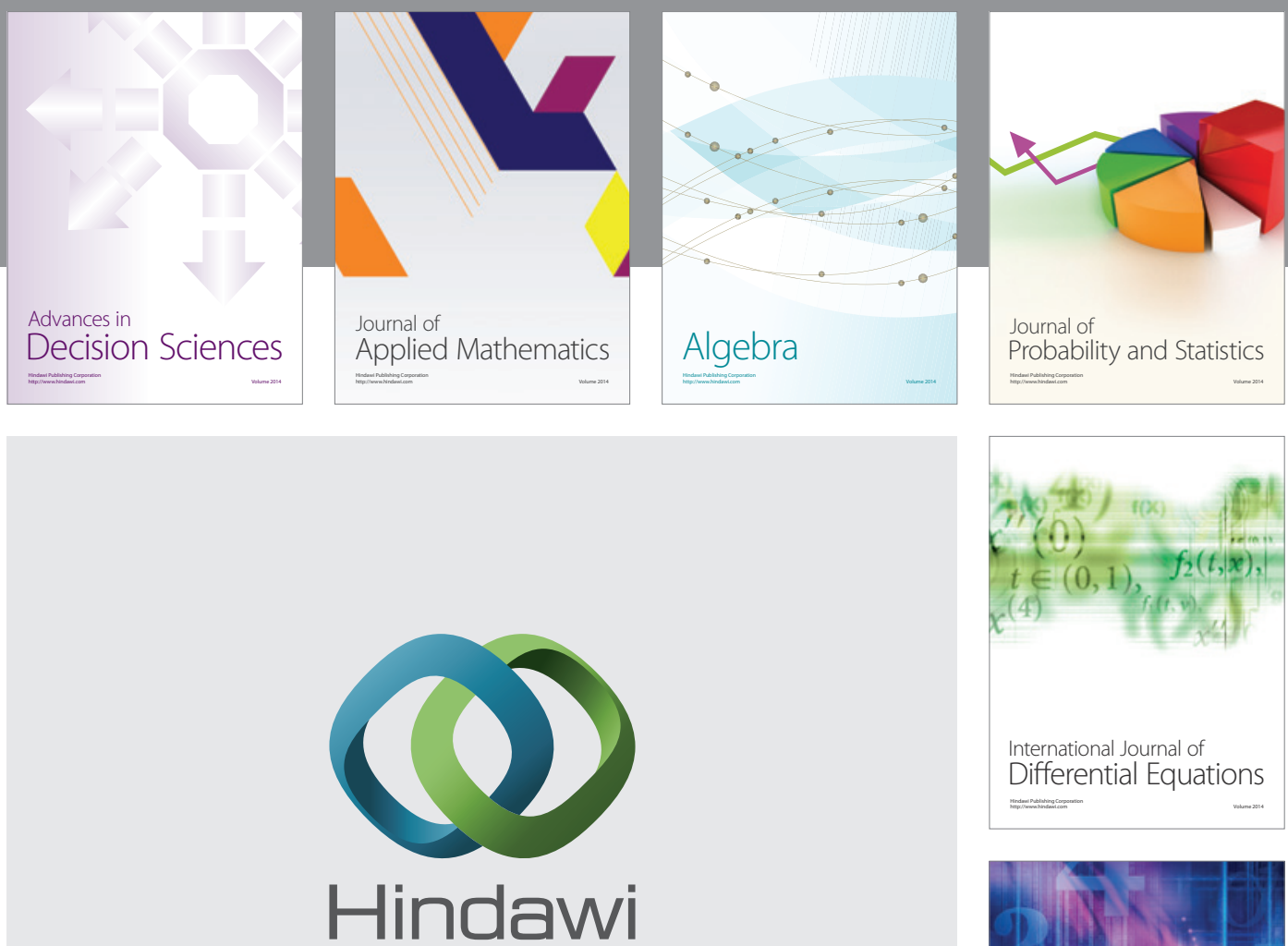

Submit your manuscripts at http://www.hindawi.com
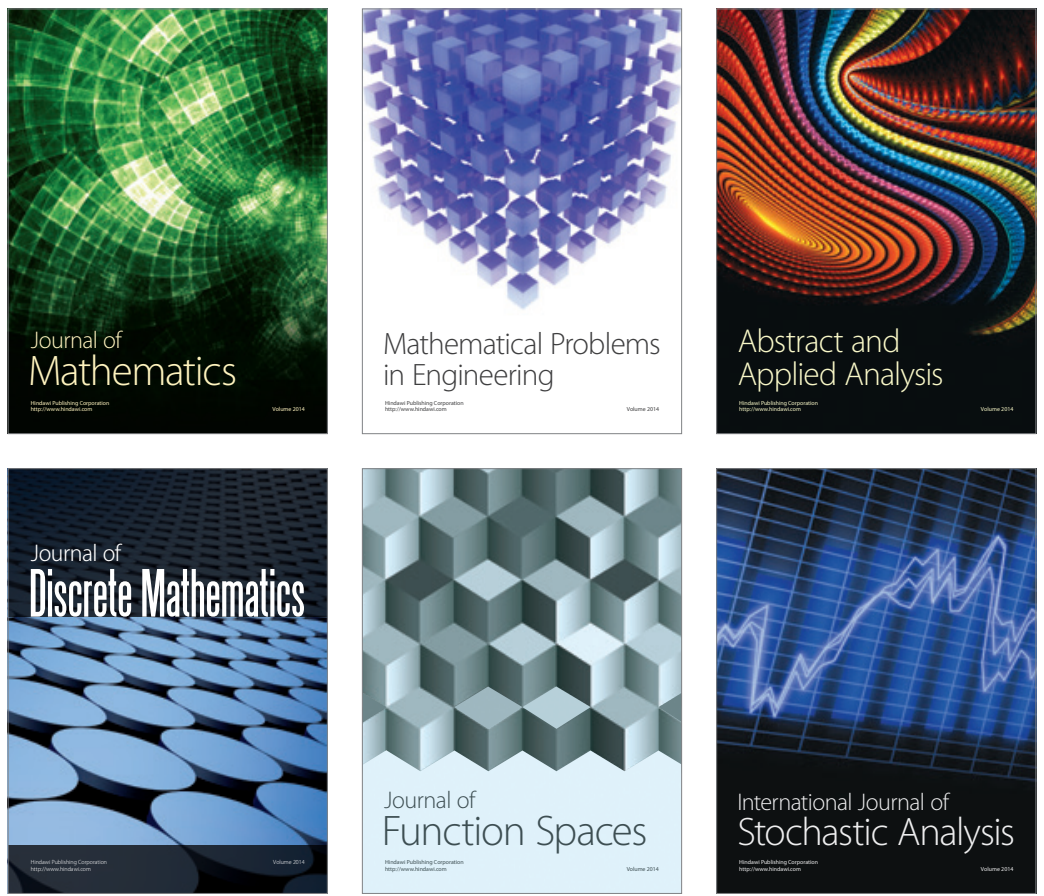

Journal of

Function Spaces

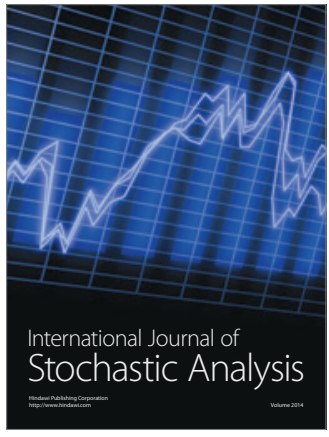

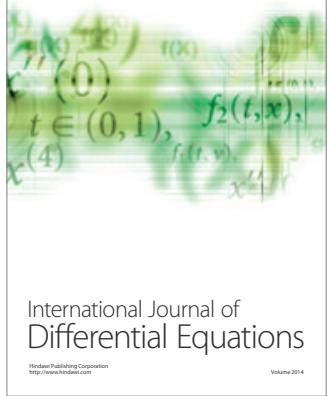
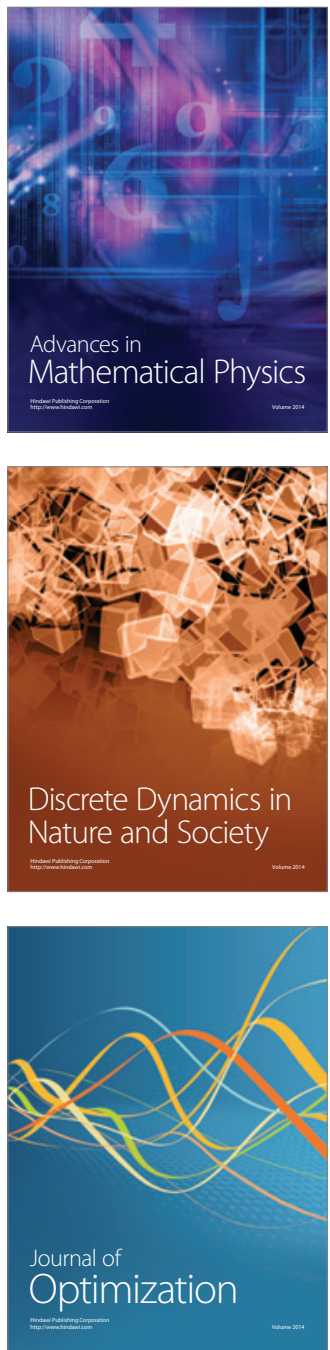